\title{
Scaling laws in turbulence
}

\author{
Christophe Josserand, ${ }^{1}$ Martine Le Berre, ${ }^{1,}$ a) and Yves Pomeau ${ }^{1}$
}

Ladhyx (CNRS UMR 7646), Ecole Polytechnique, 91128 Palaiseau, France

(Dated: 21 November 2020)

Following the idea that dissipation in turbulence at high Reynolds number is dominated by singular events in spacetime and described by solutions of the inviscid Euler equations, we draw the conclusion that in such flows scaling laws should depend only on quantities appearing in the Euler equations. This excludes viscosity or a turbulent length as scaling parameters and constrains drastically possible analytical pictures of this limit. We focus on the drag law deduced by Newton for a projectile moving quickly in a fluid at rest. Inspired by this Newton's drag force law (proportional to the square of the speed of the moving object in the limit of large Reynolds numbers), which is well verified in experiments when the location of the detachment of the boundary layer is defined, we propose an explicit relationship between Reynolds stress in the turbulent wake and quantities depending on the velocity field (averaged in time but depending on space). This model takes the form of an integro-differential equation for the velocity which is eventually solved for a Poiseuille flow in a circular pipe.

This article should have been published in the special issue of Chaos dedicated to the eightieth birthday of Professor Enrique Tirapegui, a friend and a pioneer of nonlinear science. Enrique did pass away before this special issue was completed. So we dedicate this paper to his memory in the hope that the great tradition he initiated in this fascinating field of science in Chile and elsewhere will be kept alive with the high level of quality of his outstanding research.

One long standing problem in turbulence and chaos theory is the understanding of Newton's drag law according to which the drag force on fast moving objects is proportional to the square of its velocity, with a factor of proportionality depending on the Reynolds numbers in the range of moderate values and tending to a constant in the highly turbulent regime at large Reynolds number. We argue here that this follows from the property that dissipation in fully developed turbulence (which is ultimately due to effects induced by the molecular structure of fluids), takes place at events described by singular solutions of the Euler inviscid equations.

\section{INTRODUCTION}

Turbulent flows at large Reynolds number display such a complex behavior in space and time that it is impossible to obtain a general solution of the fluid equations representing well the flow field in these situations. As recognized early by Reynolds this makes necessary a statistical description of the

\footnotetext{
a) Electronic mail: martine.le-berre@u-psud.fr; Also at ISMO (CNRS UMR 8214), Université de Paris-Saclay, 91405 Orsay, France
}

fluctuations in those flows ${ }^{1-3}$. A major issue met when developing this statistical picture is the choice of physical parameters that should enter in such a theory. Kolmogorov suggested (see section $33 \mathrm{in}^{1}$ ) to use $\varepsilon$, the dissipated power per unit mass on average, as a scaling parameter for the statistics of turbulence, assumed to be homogeneous and isotropic. In this picture, $\varepsilon$ is independent of the viscosity (see below) and the energy of the fluid motion is dissipated homogeneously and isotropically. Even though this last assumption is done very often in theoretical works, the kind of physical situations to which it applies is far from obvious. Specifically most turbulent flows, if not all of them, have a geometrical structure making them non homogeneous and non isotropic.

This obvious remark is behind the developments presented below leading to an expression of the Reynolds stress tensor in terms of the time averaged velocity field $u(X)$ depending on space only. This expression does not contain any small intrinsic length (like the mean free path in Maxwell kinetic theory). Indeed, contrary to the case of kinetic theory, there is no length scale in turbulence defined independently of the turbulence itself. Therefore an Enskog-like theory cannot be used because it yields a local relation between fluxes of conserved quantities (like mass, energy, impulse and eventually electric charge) and their gradients and higher derivatives. This leads naturally to look for a nonlinear and nonlocal relation between the Reynolds stress tensor and the strain tensor (gradient of the mean velocity, a tensor called sometimes rate of strain tensor). This relation contradicts the Boussinesq assumption made in 1875 , which states that the two tensors are aligned, the proportionality coefficient being defined as the turbulent viscosity. Even though Boussinesq did not quote Maxwell, his theory could have been inspired by Maxwell theory of the viscosity of gases, going back to 1867 . Boussinesq assumption has been proved since to be incorrect ${ }^{4}$ because the proportionality (or alignment of the two tensors) is observed neither in numerical studies nor in experiments. However, it is at the heart of most of the numerical schemes used practically, of course in more sophisticated versions, but still using the parallelism between the Reynolds stress and the strain tensors ${ }^{5}$. The reasons are firstly because of the simplicity of its numerical implementation and the numerical efficiency of the method; but also 
because the determination of numerous adjustable parameters in these models eventually allows for a good approximation of the pertinent quantities needed for application. Let us eventually notice that the non proportionality of Reynolds stress and rate of strain tensors is one of the poorly understood phenomena observed in turbulence. Among the others are

i) Newton's law of proportionality between the turbulent drag force and the square of the velocity on a blunt body moving swiftly in a fluid (at large Reynolds number in modern terms),

ii) the intermittency of the velocity fluctuations in the same range of large Reynolds numbers,

iii) the splitting of the flow around fastly moving blunt bodies into an upstream non-turbulent potential flow and a downstream turbulent wake with vorticity.

We argue in this paper that those observations can be explained by the occurrence of finite time singularities in solutions of the inviscid Euler equation. Thanks to that one can derive an integral (in space) equation relating Reynolds stress and the average velocity field. The irreversibility in the dynamics of the Euler velocity is the result of finite time singularities of this Eulerian dynamics. In such singularities energy is likely dissipated not by viscosity but instead by effects in the Enskog expansion at the order following the regular viscous term. However the amount of energy dissipated is the one present initially in the collapsing domain and so is given by the inviscid dynamics only.

The existence of singularities, a very hard problem initiated by Leray in $1934^{6}$, is the key point of our study. It concerns the dynamics of the Euler equations with initial conditions of finite energy. Let us recall briefly some points in favor of their existence in turbulent flows. By analyzing time records of the velocity in the big wind tunnel of Modane, it has been found ${ }^{7,8}$, we believe, convincing evidence that the occurrence of such singularities explains well the observed intermittency of the signal. Our proof is based on the following arguments. Kolmogorov scaling assumption agrees with the von Karman-Howarth relation ${ }^{1}$ for the triple velocity correlation. It amounts to assume that, by neglecting the term of viscosity in the NS equations, a velocity fluctuation $\delta u$ evolves at typical distance $\delta r$ like the cubic root of this distance, namely that $\delta u \sim(\varepsilon \delta r)^{1 / 3}$. Taking the cube of both sides of this scaling relation, one gets the von Karman-Howarth relation. However this scaling law does not seem to be consistent with the Euler equations for an incompressible flow. This follows from a simple estimate of the local acceleration, this acceleration having the same order of magnitude as $u \nabla u$. Using the Kolmogorov scaling one finds $\nabla u \sim \delta u / \delta r$, which gives a local acceleration of order $\bar{u} \varepsilon^{1 / 3} \delta r^{-2 / 3}$, where $\bar{u}$ is the local value of the velocity. This is inconsistent with the fact that the acceleration is the time derivative of the velocity because at short times $\Delta t$ the increment of the velocity derived from the acceleration is of order $\Delta t$ times the time derivative of $u$ just estimated. This adds to the velocity $u$ a quantity of order $\Delta t \bar{u} \varepsilon^{1 / 3} \delta r^{-2 / 3}$, far bigger as $\delta r$ tends to zero than the estimate $\delta r^{1 / 3}$ one started from, which was consistent with a continuous function of Hölder exponent 1/3: the new Hölder exponent is negative now. This large fluctuation of the ac- celeration is more than a mathematical bug evidenced by this calculation, it is also something that is seen, although quite indirectly, in records of velocity fluctuations in a high speed wind tunnel. There, contrary to what is implied by the estimate $\delta u \sim(\varepsilon \delta r)^{1 / 3}$, the large fluctuations of acceleration do not happen all the time, but are strongly correlated to sparse large velocity fluctuations ${ }^{7}$. Such a correlation contradicts Kolmogorov scaling law because in this scaling law the parameter $\varepsilon$ is just the product of the velocity and of the acceleration. Had this parameter a fixed value, or at least without too large fluctuations, large velocities should be correlated to small accelerations and conversely large accelerations correlated to small velocities, exactly the opposite of what is observed.

Therefore it seems meaningful to reconsider the scalings laws for turbulence. It is important here to notice that, by itself, large gradients of the velocity field are not proofs of the existence of singular or quasi singular events. Consistent with the scaling law $\delta u \sim(\varepsilon \delta r)^{1 / 3}$, a possibility could be that the velocity field is a Weierstrass-like function of the position with Hölder exponent $1 / 3^{9}$. This would make the velocity field continuous but not derivable, whence it does not show a priori the observed correlations between large gradients of the velocity and large acceleration in singular points in space and time. The correlations between large velocities and large accelerations instead is a witness of the existence of point-like singularities. Another possibility for the dependence of the velocity field as a function of position and time was considered in 1934 by Leray ${ }^{6}$. It assumes that the solutions of the fluid equations display finite time singularities that are also localized in space points. Here we start from this assumption, supported by our recent investigations of turbulent flows. The strong correlation between large values of the velocity $\mathbf{u}$ and of the acceleration $\gamma=\partial_{t} \mathbf{u}$, at the same point in space-time are explained by the occurrence of Leray like singularities of the velocity filed in the form:

$$
\mathbf{u}(\mathbf{r}, t)=\left(t^{*}-t\right)^{-a} \mathbf{U}\left(\mathbf{r}\left(t^{*}-t\right)^{a-1}\right),
$$

where $t^{*}$ is the time of the singularity, and $a$ is a positive exponent smaller than unity. We have shown ${ }^{7}$ that $a$ can take the values $1 / 2$ and $3 / 5$ corresponding respectively to self-similar solutions which conserve the circulation and the energy in the singular domain.

For such solutions the space domain of large fluctuations shrinks with a (positive) power of $\left(t^{*}-t\right)$, the time difference between the time of measurement and the time of blow-up. Moreover the amplitudes of velocity and of the acceleration diverge with respect to this time difference according to power laws. Another element in favor of the occurrence of finite time singularities is the behavior of the structure function for the Eulerian acceleration,

$$
\mathscr{S}_{n}(r)=<\delta \gamma^{n}>
$$

where $\delta \gamma=\gamma\left(r+r_{0}\right)-\gamma\left(r_{0}\right)$. When the order $n$ in $\mathscr{S}_{n}(r)$ changes, the behavior of this function of $r$ changes dramatically $^{8}$. At small order the structure function built with the acceleration tends smoothly to zero as the distance $r$ between 
its two arguments tends to zero whereas it diverges at small distances when the order is larger than a critical value. This change of behavior of $\mathscr{S}_{n}(r)$ (with respect to $n$ ) is a straight consequence of the existence of singularities. This property results from the calculation of $\mathscr{S}_{n}(r)$ as an integral over the space-time volume of $\delta \gamma^{n}$ weighted by the probability of recording a singularity at a given point of space-time, which is small at small distance. We showed that the structure function itself depends on $r$ at small values like $r^{p(n)}$, with the structure exponent $p(n)=a_{1}-a_{2} n$ on $n$ with a positive constant $a_{1}$ added to a linear term of negative coefficient $-a_{2}$. When $n$ is less than a critical value the result of the integration defining the structure function tends to zero at small distances, but when the exponent of the structure function becomes bigger than a critical value, the divergence of amplitude of the singularity overcomes the small probability of observing a singularity so that the structure functions become divergent at small distances. The same non trivial behavior of the structure function is observed in a nonlinear-Schrödinger model equation which displays numerically turbulent solutions and quasi-singular events, in a limit equivalent to the one of zero viscosity ${ }^{10}$. Furthermore the structure functions of acceleration recorded in wind tunnels at large distances $r$ tend to a constant because they represent contributions of quasi independent singularities. Of course a very significant question is the mathematical proof of existence or absence of singular solutions of the equations of Leray-like singularities for the dynamical Euler equations (what we call the Euler-Leray equations). This has been the matter of many investigations since the time of Leray publication. We can only mention some progress made recently ${ }^{8}$ toward an explicit construction by perturbation of a solution of the Euler-Leray equations, not yet complete. The idea is to start from a family of explicit non trivial solutions of the steady Euler equations in an axisymmetric geometry with swirl. This makes a solution of part of the Euler-Leray equations. The remaining of those EulerLeray equations can be treated in some limit as a small perturbation. This leads to solutions of the Euler equations slowly collapsing to a singularity. What remains to be solved is a pair of solvability conditions in the expansion. Those conditions constrain the choice of the base solution of the Euler equation one starts from. This step is non trivial because it depends on kernels of non symmetrical linear operators, a priori unknown and hard to find numerically. On the positive side this leads to two solvability conditions whereas there is a continuum of different steady solution of the Euler equation, which depend on an arbitrary polynomial, so that one can hope that with a right choice of the arbitrary coefficients of this polynomial the two solvability conditions (implying the cancelation of scalar products of functions) can be satisfied.

There remains another non trivial question in fluid mechanics, which is the effect of a small viscosity on those singular events. It is widely believed that at small scales of turbulence viscosity becomes dominant. As a self-similar solution collapses, the space scale tends to zero at this point, that makes the viscous stress bigger and bigger as the singularity time is neared, but at the same time the velocity fluctuation becomes larger and larger. So it may happen that this velocity increase overcomes the increase of viscous stress due to the shrinking of length scales. This is exactly what happens in the Leray equations in the case $a=3 / 5$ which corresponds to finite energy conserved in the shrinking domain until viscosity (or Burnett coefficients) become effective. We showed that in this case the perturbation of Euler dynamics due to viscosity diverges less strongly than the non linear terms as one approaches the singularity, so that the viscous pressure becomes a negligible perturbation near the blow-up time. Therefore, at least in this case, there is no guarantee that viscosity regularizes the solution at the singularity time. We refer the interested reader to the original publication on this question ${ }^{8}$, the conclusion being that dissipation takes place always near the singularity time but not necessarily because of the effect of viscosity. To summarize this discussion, the "natural" evolution of solutions of the fluid equations is toward finite time singularities where velocity gradients are cancelled, that ultimately explains Newton's drag on fast moving bodies. As shown below this helps to derive an explicit and closed set of equations for the average velocity field, that finally yields a dissipation term independent of the viscosity.

\section{EXPRESSION OF REYNOLDS STRESS}

From what was concluded at the end of previous section, dissipation in highly turbulent flows takes place in Leray-like singularities of solutions of the Euler dynamical equations. In fact, they cannot be real singularities in continuous media like fluids and those singularities are smoothed out by some sort of correction to the Euler equations taking into account the molecular structure of the fluid. Usually the role of this regularizing effect is played by viscosity, but as just said and reconsidered in the next subsection in the light of drag forces, it is not necessarily true that viscosity cuts the singularity before blow-up. As shown in reference ${ }^{8}$, higher order terms in the Enskog expansion- in an extended form- do smoothen the singularity in the case $a=3 / 5$. The precise way they do it is not relevant here. It relies on higher order terms in the Enskog expansion, the so-called hyper viscosity Burnett terms, or more accurately the "renormalized Burnett terms", since the coefficients of those terms are given by diverging Green-Kubo integrals. Then the hyper viscosity contributions to stress in the fluid equations have to be renormalized in order to include non-local integrals (or if one prefers non integer Liouville space derivatives). The next step in this theory is to find what is the effect of such singularities on the global fluid dynamics averaged over time. In this section, we investigate the role of singular events on the drag felt by objects moving at large Reynolds number, the singular events being the main source of dissipation.

\section{A. Drag force}

At the very beginning of fluid mechanics, as we know it, was the law of drag by Newton for fast moving projectiles in air. By a very clever reasoning Newton showed that the 
drag force on an object moving at speed $U$ in a fluid at rest at infinity is of order

$$
F^{(N)}=\frac{1}{2} C_{x} \rho U^{2} S,
$$

where $\rho$ is the mass density of the fluid and $S$ the area of the cross section of the moving object in the direction perpendicular to the velocity. Lastly $C_{x}$ is a numerical coefficient which, to Newton's surprise, depends on the shape of the object in a complex way that he was unable to explain. As well known too, d'Alembert showed that in a perfect inviscid fluid there is a steady flow around a moving object exerting no drag on it (see sections 9 and 11 in $\operatorname{ref}^{1}$ ). In the familiar example of a sphere moving at constant speed, Euler's equations can be solved explicitly and d'Alembert paradox applies so that no drag should be exerted on the sphere. Neither Newton nor d'Alembert refer to turbulence, a concept unknown at their time. The resolution of d'Alembert paradox is that dissipation takes place in the wake of the moving object and yields ultimately a drag in agreement with Newton's insight. In retrospect Newton's law for the drag is an early version of what is called sometimes the zeroth law of turbulence, namely that, in the limit of high Reynolds number, dissipation tends to a value that does not depend on viscosity (this amounts to say that, in the limit of large Reynolds number, Newton's $C_{x}$ coefficient tends to a constant, something in full agreement with observations $^{3}$ ). A perhaps less well known law of turbulent friction (in modern terms) in high velocity fluids is due to Chézy ${ }^{11}$. It goes back to the mid eighteenth Century and relates the velocity of a river flow to the slope and the cross section of this river. It relies on a friction proportional to the velocity square and is in fair agreement with field data, up to a coefficient (the Chézy coefficient of order one) depending on the roughness of the river bottom. Similar friction laws were written down later for pipe flows by Prandtl and his school, with particular consideration of the dependence of the friction coefficient with respect to the roughness of the pipe wall ${ }^{12}$.

The connection between the drag law of Newton and our search of scaling laws for fully developed turbulence comes from the fact that, physically, Newton's drag represents the dissipation by turbulence in the wake of a fast moving body. Let us first note that the dissipation by viscosity in the viscous sublayer in front of a moving body is much less than the dissipation given by Newton's drag law, since the ratio of these two quantities is of order $R e^{-1 / 2}, R e$ being the Reynolds number, as it follows from Prandtl scaling for boundary layer theory. Moreover let us also show that the drag force due to the laminar boundary is proportional to the power $3 / 2$ of the velocity whereas the one due to the turbulent wake (Newton's drag) is proportional to the velocity square, that makes it dominant at large Reynolds number. Therefore dissipation in the laminar boundary layer is relatively negligible at large Reynolds number. Because this remark is hard to find in the literature of fluid mechanics, we are going to compare Newton's drag and drag in the laminar boundary layer.

Newton's drag law can be derived as follows. Suppose a body of cross section $S$ moving at velocity $U$ in a fluid at rest of mass density $\rho$. Per unit time the body crosses a cylinder of fluid of mass $\rho S U$ and gives it the velocity $U$. Therefore the momentum given to the fluid per unit time is this mass multiplied by the velocity $U$, and this is also the drag force because of the conservation of momentum, the second law of Newtonian dynamics. This yields a drag force proportional to $\rho S U^{2}$, as just stated. Newton specifies that this law is correct for fluids "without tenacity", which may include perfect fluids. A possible understanding of "tenacity" is that adjacent layers of fluid tend to move at the same speed, which makes tenacity more or less equivalent to the property typical of viscous fluids. .

The derivation of the drag due to dissipation in the laminar boundary layer is less direct than the one of Newton's law. This derivation considers the contribution of the viscous friction force to the drag force in a laminar boundary layer close to the body. Let $L$ be the typical length of this layer along the surface of the body. In the boundary layer the tangential velocity is of order $U$. Over a length $L$ along the surface, viscosity makes diffuse the vorticity on a length scale $\delta$, the thickness of the boundary layer. The constant of diffusion is $v=\frac{\mu}{\rho}$, therefore the thickness is $\delta=(v L / U)^{1 / 2}$ where the equal sign is to be understood as meaning same order of magnitude. This thickness enters into the estimate of the viscous stress as $\sigma=\mu U / \delta$. From its meaning this stress yields a total force of friction equal to $\sigma L^{2}$, assuming that the size of the boundary layer is about the same in the directions parallel and perpendicular to the speed.

Combining those estimates one finds that the friction $F_{b l}$ due to the boundary layer is

$$
F_{b l}=D U^{3 / 2} \rho^{1 / 2} \mu^{1 / 2} L^{3 / 2} .
$$

where $D$ is a dimensionless coefficient, presumably of order one.

Comparing this with Newton's law of drag one sees that Newton's drag is about $F_{b l}$ times the square root of the Reynolds number $\rho U L / \mu$. Therefore this contribution of the laminar boundary layer is relatively negligible in the limit of a large Reynolds number. It could be argued that in the limit of large Reynolds number this boundary layer becomes turbulent so that the order of magnitude of the friction there is much bigger than what is predicted by the above estimate. It is certainly correct that at very large Reynolds number the boundary layer becomes turbulent but, in this case, is seems hard to distinguish between the contribution of the turbulent wake and the one of this turbulent boundary layer to the total drag, the later being part of the contribution of the turbulent wake.

\section{B. Beyond Boussinesq relation for the stress tensor}

Let us start from the Reynolds-averaged Navier-Stokes equations for a statistically stationary flow of a Newtonian incompressible fluid which can be written in Einstein notation and Cartesian coordinates as

$$
\rho \bar{u}_{j} \frac{\partial \bar{u}_{i}}{\partial x_{j}}=\frac{\partial}{\partial x_{j}}\left[-\bar{p} \delta_{i j}+\mu\left(\frac{\partial \bar{u}_{i}}{\partial x_{j}}+\frac{\partial \bar{u}_{j}}{\partial x_{i}}\right)-\rho \overline{u_{i}^{\prime} u_{j}^{\prime}}\right],
$$


where $\bar{u}$ and $u^{\prime}$ represent the time averaged and fluctuating part of the velocity field respectively, $\rho$ the density and $\mu$ the shear (or dynamical) viscosity of the fluid. Moreover the pressure $p$ is derived from the condition that the velocity field is divergenceless. In equation (4) the change in mean momentum of fluid element (left term) is balanced by the isotropic stress owing to the mean pressure field $\bar{p}$ and the viscous stress

$$
\sigma_{i j}^{v}=-\mu\left(\overline{u_{j, i}}+\overline{u_{i, j}}\right),
$$

where $u_{i, j}=\frac{\partial u_{i}}{\partial x_{j}}$, and the nonlinear Reynolds stress

$$
\sigma_{i j}^{R e}=\rho \overline{u_{i}^{\prime} u_{j}^{\prime}}
$$

which requires additional modeling to close (4) for solving, and has led to many different turbulence models ${ }^{5,12}$.

Almost all of them start with the empirical Boussinesq hypothesis which amounts to assume that the turbulent or Reynolds stress tensor $\sigma_{i j}^{R e}$ is aligned with the strain or viscous tensor $\sigma_{i j}^{v}$, namely that

$$
\overline{u_{i}^{\prime} u_{j}^{\prime}}=-v_{t}\left(\overline{u_{j, i}}+\overline{u_{i, j}}\right)
$$

where $v_{t}$, called turbulent kinematic viscosity has the dimension of a velocity times a length. The Boussinesq relation (7) with constant $v_{t}$ is truly not adapted to describe wakes behind an obstacle or in the vicinity of frontiers like the bottom of a river or the boundary of a pipe. Boussinesq proposes to express the turbulent viscosity by an (implicit) analogy to kinetic gas theory (but for velocity), that precludes the relation formalized in 1925 by Prandtl for the turbulent viscosity as the product of a mixing length $l_{m}$ by a characteristic velocity defined by the ratio of this length with a time which is the square root of the trace of the strain tensor, $\left(\overline{u_{k, l}} \overline{u_{l, k}}\right)^{1 / 2}$, leading to the following expression for the Reynolds stress

$$
\overline{u_{i}^{\prime} u_{j}^{\prime}}=-l_{m}^{2}\left|\overline{u_{l, k}} \overline{u_{k, l}}\right|^{1 / 2}\left(\overline{u_{i, j}}+\overline{u_{j, i}}\right) .
$$

This is the so-called mixing length model where the mixing length $l_{m}$ has to be adjusted to the geometry of the flow. In this approach, the square-root term in the turbulent viscosity is often explained by an analogy between the turbulent fluctuations and the thermal fluctuations responsible of the microscopic viscosity. This model is able for instance to reproduce the Moody diagram for the friction coefficient $C_{x}$ in (3) for a parallel flow channelling along a wall (note that in this derivation the friction is assumed to occur in the boundary layer and the velocity $U$ is not the velocity of the flow, but its average in the boundary layer, perpendicularly to the wall). However this model, based on nonlinear polynomial local equations like (8) fails to reproduce more complex turbulence behaviors, as for instance in annular flows fro which both the strain and vorticity tensors vanish near the central position, whereas the Reynolds stress does not.

The scaling laws for the representation of the turbulent wake, which is the matter of this study, should be consistent with the final result, namely that the drag is independent of the viscosity. Therefore this drag must depend only on quantities occurring in the inviscid equations. This is fully consistent with the idea of dissipation by finite time singularities, because the amount of energy in such a singularity is independent of viscous phenomena and is dissipated at the singularity time. This leaves few parameters to model the turbulent flow responsible of the drag. As we are looking for time-averaged quantities one needs to look at equations for averaged quantities only, without time dependence. Compared to the standard fluid equations, there is no obvious constraint imposing that the equations we are looking for are local in space, namely written with space derivatives of finite order at a given point. This allows to write equations with integral terms, in agreement with the idea that singular events occurs here and there in the flow. Let us look at the possibility of a new term with an integral kernel and the same physical dimension as the $u \nabla u$ term in Euler equation for momentum. Because this term should be the gradient of Reynolds turbulent stress, this stress has to be quadratic with respect to the velocity (see section 15 in $\left.^{1}\right)$, as in (8).

In the following, we shall drop the bar over the timeaveraged velocity because we only consider products of $\bar{u}$, not averages of products. The simplest integral relation could be to insert into the integral the term $\left(u_{i, j}+u_{j, i}\right)$ times $|u|$ by analogy with Maxwell expression for the shear viscosity of gases, the absolute value of the fluid velocity playing there the role of the thermal velocity of particles. Inded, a fundamental result of kinetic theory is that the shear viscosity of a gas is proportional to a quantity with the physical dimension $|u| \ell$, where $|u|$ is the typical thermal speed of a particle, and $\ell$ the typical mean free path. The mean free path is the distance over which the momentum difference between two particles is carried, ending with the annihilation of this momentum difference by their collision and to the release of thermal energy. By analogy one could expect that in a singular Leraylike event, an initial difference of momentum between parts of fluid participating to the collapse is cancelled when the velocity field of the domain shrinks to zero, which erases the initial momentum difference, and so participate to the transfer of momentum in space. The role of those parts of the fluid with different velocities is played in Maxwell's theory of a dilute gas, by the two particles on their path towards a collision (which explains ultimately the shear viscosity of a dilute gas). Here, if the square root of the turbulent fluctuations $|u|$ can be invoked by analogy with Maxwell arguments, we have to explain how such non analytic behavior can arise from the Euler dynamics. While for explaining viscosity it comes either from kinetic theory for dilute gases or from the Green-Kubo relations for arbitrary densities, we want to argue below that such non analytic expression could be a consequence of the singularity dynamics of the Euler equation.

To somehow "multiply" the combination $|u|\left(u_{i, j}+u_{j, i}\right)$ by a length one has to integrate it with respect to the position variable but by keeping the requested invariance of the result under rotation of the system of coordinates. This excludes for instance to carry an integration with respect to only one space variable because the choice of one coordinate obviously breaks the symmetry under rotation of the system of coordinates. A straightforward convenient expression is found by integrating $|u|\left(u_{i, j}+u_{j, i}\right)$ computed at $X^{\prime}$ (capital letters being for position in space, $X=(x, y, z))$ over the whole space 
with the integrand $\frac{1}{\left|X-X^{\prime}\right|^{2}}$, the power of the denominator being chosen to give the whole transformation the scaling of a length. The final result is

$$
\sigma_{i j}^{R e}(X)=K \rho \int \mathrm{d} X^{\prime} \frac{\left|u\left(X^{\prime}\right)\right|\left(u_{i, j}+u_{j, i}\right)\left(X^{\prime}\right)}{\left|X-X^{\prime}\right|^{2}},
$$

where $K$ is a pure number, independent on the chosen system of units. In this expression the velocity has to be measured with respect to a frame of reference, which can only be the one of the object moving in such a way that it makes the flow turbulent. However the relation (9) for Reynolds stress does not satisfy the principle of Galilean invariance, although it is compatible with the requirement that no parameter other than what appears in Euler equation is introduced in the phenomenological relation between the average velocity field and Reynolds stress. The expression (9) applied to the case of a Poiseuille flow in a circular pipe allows to get a solution for $u(r)^{2}$ up to a constant, that is different from the expected result $(u(r) \text { up to a constant velocity })^{13}$. It appears therefore that the Reynolds stress must contains space derivatives only, as in the Prandtl model (8).

Within the same constraints another choice can be made. It is based upon the idea that out of the velocity field $u(X)$ one can build another vector field, the vorticity $\nabla \times u$. This vector field can be substituted to the one appearing in this equation inside the modulus, which amounts to replace $\left|u\left(X^{\prime}\right)\right|$ by $\left|\nabla \times u\left(X^{\prime}\right)\right|$ in this equation. Of course this change needs to be compensated by a multiplication by $\left|\left(X^{\prime}-X\right)\right|$ to keep the property that the contribution to the Reynolds stress tensor (divided by $\rho$ ) has the physical dimension of a velocity square without dependence with respect to the unit length. The final result amounts to the following form of the Reynolds stress

$$
\sigma_{i j}^{R e}(X)=K \rho \int \mathrm{d} X^{\prime}\left(\frac{\left|\nabla \times u\left(X^{\prime}\right)\right|}{\left|X-X^{\prime}\right|}\right)\left(u_{i, j}+u_{j, i}\right)\left(X^{\prime}\right) .
$$

Another possible formulation the Reynolds stress should be an expression more symmetrical with respect to the coordinates $X$ and $X^{\prime}$. Such a symmetry is obtained by putting $\left|\nabla \times u\left(X^{\prime}\right)\right|^{1 / 2}|\nabla \times u(X)|^{1 / 2}$ instead of $\left|\nabla \times u\left(X^{\prime}\right)\right|$. More generally we will eventually propose a Reynolds stress based on this product but using dexponents $\alpha$ and $1-\alpha$ for the different vorticity terms (see formula 13). This balanced dependence of the vorticity in $X$ and $X^{\prime}$ could fulfill the requirement that vorticity must be re-amplified, a fundamental property of turbulent flows. Let us recall that Reynolds saw turbulence as this mechanism of amplification of vorticity, which has to be included in the tools used for its description, if one wants to go beyond the d'Alembert paradox.

A point of interest is the assumption behind the occurrence of the absolute value $|u|$ and $|\nabla \times u|$ in the expressions (9) and (10) of the Reynolds stress tensor. Indeed this is not analytical with respect to the velocity field and so cannot be derived from Euler's equation by simple operations on Euler's equation which preserve the analyticity of the result with respect to the velocity. Reynolds stress tensor is derived as a time average of the product of velocities. This time average extends itself to all kind of events occurring in the fluid dynamics. In our view of turbulence we include finite time Leray-singularites of Euler equation. When the time average includes such singular events, there is no reason to impose the result to depend analytically on the velocity field. This explains the origin of this non analyticity of our form of Reynolds stress tensor with respect to the velocity field because of the absolute value appearing in it. This shows also that it cannot be derived from Euler equation by a calculation using standard algebra. The physics of this law is that finite-time singularities dissipate energy and so introduce a fundamental irreversibility in solutions of equations (the Euler equations) which are formally reversible with respect to time. Deriving irreversible equations from reversible dynamics is fairly standard in theoretical physics, the most famous example being the derivation of Boltzmann kinetic theory from the reversible Newtonian dynamics of particles. In the present case, the irreversibility is a consequence of the dissipation taking place at the time of the singularity of the self-similar solution of Leray-like equations. As shown in reference ${ }^{7}$ in the case of a turbulent Poiseuille flow, the irreversibility of the equation is tightly linked to the absolute value in the equation for the turbulent stress because thanks to it, the average flow in a pipe is reversed as the pressure gradient is reversed. The absolute value is a consequence, although slightly hidden, of the existence of dissipation in singular events. Otherwise (if the equation for the turbulent stress does not contain absolute value of the velocity) there is no dissipation by the Euler equation. This explains, hopefully, why equations (9)-(10) cannot be derived directly from Euler equation by assuming their solution to be smooth. An added information is necessary, namely that solutions of Euler equations have finite time singularities where energy is dissipated.

A last important remark is about the question of how general is equation (10). This equation for Reynolds turbulent stress was derived under the constraint that this stress may depend only on parameters of the average velocity field, without introducing any new quantity with a physical dimension like a length or a time scale. This is compatible with a change of the equation preserving the physical dimension of the stress. Such a change could be made by multiplying the stress by a non trivial dimensionless combination built with the average velocity field and its derivatives. Such a dimensionless combination is the ratio

$$
R a=\frac{u_{i, j} u_{j, i}}{|\nabla \times u(X)|^{2}}
$$

This ratio is invariant under the elementary change of reference frame by rotation, translation and Galilean transform, as well as by arbitrary dilation of $u$ and of length scale. Moreover $R a$ takes different values according to the type of the velocity field (assumed to be divergenceless), for instance an uniform rotation yields $R a=0$ because the numerator cancels, whereas a uniform shear field yields a non zero constant $R a$. It is not too hard to write the general expression of $R a$ as a function of the first derivatives of the incompressible velocity field at one point. Because $R a$ is a dimensionless ratio (depending on $X$ ), any numerical function of it may multiply the right hand side of the equation for the Reynolds stress as a function of the average velocity field by keeping the constraints of Galilean and 
frame-invariance together with the independence with respect to an a priori defined length scale.

Other combinations than $R a$ may also be built from the velocity field by keeping the same symmetries. One can replace the numerator $u_{i, j} u_{j, i}$ by the higher order polynomial $u_{i, j} u_{j, k} u_{k, i}$ or its extensions to any power of the gradient of the velocity field. Once divided by the right power of the modulus of the curl of $u$ one obtains a scalar depending in a dimensionless way of the average velocity field and its derivatives

$$
R b=\frac{u_{i, j} u_{j, k} u_{k, i}}{|\nabla \times u(X)|^{3}} .
$$

Moreover, because $R a, R b$ and their higher order kins are dimensionless scalars one can put them in the argument of numerical functions substituting the numerical constant $K$ with a result that is a quantity keeping the invariance properties of $R a, R b$, etc. Multiplying equations (10) for Reynolds stress by such functions leaves a lot of freedom for modeling turbulent flows with a more or less complex structures.

The equation for the average velocity in the turbulent flow is a time-independent equation derived by adding Reynolds stress to the term $u_{i} u_{j}$ in the equation for the time averaged Euler equation for the momentum. This yields

$$
\partial_{i}\left(\rho u_{i} u_{j}+\sigma_{i j}^{(R e)}\right)+\partial_{j} p=0,
$$

The condition of incompressibility $\frac{\partial u_{i}}{\partial x_{i}}=0$ is to be added to equations (10) and (12) in order to derive the spatial dependance of the time averaged velocity $u(X)$. The applicability of equation (10) to given situations like the turbulent flow in a pipe is dealt with in next section. It shows that in the case of pipe flows as well as in other cases like flows in $2 \mathrm{~d}$ geometries the integral in this equation diverge logarithmically. The convergence of this integral is looked at in the following section, and a solution is proposed in subsection III D to bypass this difficulty without introducing an arbitrary cut-off at large distance.

\section{POISEUILLE FLOW IN A PIPE}

An example showing how to use these equations for specific problems of fluid mechanics, is the case of Poiseuille flow in a pipe with the axis along the coordinate $x$. For plane or circular Poiseuille flow, the $x$-component of the velocity is the only one which is non-zero and it depends on $(y, z)$ only. Therefore the only non-vanishing components of $\sigma_{i j}^{R e}$ in (13) are $\sigma_{x y}^{R e}(y, z)$ and $\sigma_{x z}^{R e}(y, z)$. From the symmetry of the stress tensor, two other components are non zero, one has $\sigma_{x y}^{R e}=\sigma_{y x}^{R e}$ and $\sigma_{x z}^{R e}=\sigma_{z x}^{R e}$.

For a pipe of circular section, the various quantities involved depend on the radial distance only, $r=\left(y^{2}+z^{2}\right)^{1 / 2}$ and the non vanishing component of the Reynolds stress written with the cylindrical coordinates $(x, r)$ is $\sigma_{x r}^{R e}(r)$. Our aim is to derive the mean velocity profile $u(r)$ testing the different expressions of $\sigma_{i j}^{R e}$ introduced above.

An obvious remark about the above expression of the Reynolds stress is that it is Galilean invariant, which means that by adding an arbitrary constant velocity to a solution one gets the same value of the stress tensor. For a flow around a blunt body this Galilean transform does not yield another solution, because it is not compatible with the boundary condition on the surface of the body, namely that the normal velocity is zero on this surface. This is clearly different for a pipe flow without viscosity where addition of a constant velocity parallel to the axis of the pipe is permitted and is compatible with the boundary condition for an inviscid fluid since the tangential velocity is arbitrary on a solid. But this is not the case anymore for a viscous fluid, which ultimately explains why turbulence in pipe flows is special, because of the existence of boundary layers on the walls. This is discussed at the end of this section.

\section{A. flow in finite length pipe}

In the turbulent regime with strong vorticity, dropping the potential term in (10), and balancing the vorticity between the space points $X$ and $X^{\prime}$, that is more general than in (10), we assume that the Reynolds stress is of the form

$\sigma_{i j}^{R e}(X)=K \rho|\nabla \times u(X)|^{1-\alpha} \int \mathrm{d} X^{\prime} \frac{\left|\nabla \times u\left(X^{\prime}\right)\right|^{\alpha}}{\left|X-X^{\prime}\right|}\left(u_{i, j}+u_{j, i}\right)\left(X^{\prime}\right)$,

where the exponent $\alpha$ is such that $|\alpha|<1$. We consider a pipe with circular cross section of radius $R$, aligned along the $x$-direction. Let $u$ be the x-component of the velocity. The various quantities involved depend on the radial distance only, $r=\left(y^{2}+z^{2}\right)^{1 / 2}$ and the non vanishing component of the Reynolds stress written with the cylindrical coordinates $(x, r)$ is

$$
\begin{aligned}
\sigma_{x r}^{R e}(r)= & K \rho\left|u_{, r}(r)\right|^{1-\alpha} \int_{0}^{R} \mathrm{~d} r^{\prime} r^{\prime}\left|u_{, r^{\prime}}\left(r^{\prime}\right)\right|^{\alpha} u_{, r^{\prime}}\left(r^{\prime}\right) \int_{-\infty}^{+\infty} \mathrm{d} x^{\prime} \\
& \times \int_{0}^{2 \pi} \mathrm{d} \varphi \frac{1}{\left(r^{2}+r^{\prime 2}-2 r r^{\prime} \cos (\varphi)+x^{\prime 2}\right)^{1 / 2}} .
\end{aligned}
$$

In the simple case of a long pipe of length $2 L$, with $L>>$ $R$, the calculation at $x=0$ (in the middle of the pipe) can be performed analytically. Defining $a^{2}\left(r, r^{\prime}, \varphi\right)=r^{2}+r^{\prime 2}-$ $2 r r^{\prime} \cos \varphi$. the integral over $x^{\prime}$ is

$$
\begin{array}{r}
I\left(\varphi, r, r^{\prime}\right)=\int_{-L}^{+L} \mathrm{~d} x^{\prime} \frac{1}{\left(r^{2}+r^{\prime 2}-2 r r^{\prime} \cos (\varphi)+x^{\prime 2}\right)^{1 / 2}} \\
=\ln \frac{1+\sqrt{(a / L)^{2}+1}}{-1+\sqrt{(a / L)^{2}+1}},
\end{array}
$$

The integral over $\varphi$ of $I\left(\varphi, r, r^{\prime}\right)$ gives a function depending on $r$ and $r^{\prime}$,

$$
\mathscr{I}\left(r, r^{\prime}\right)=2 \int_{0}^{\pi} \mathrm{d} \varphi I\left(\varphi, r, r^{\prime}\right)
$$

Numerically we find that as $r / R$ approaches unity, the curves $\mathscr{I}\left(r, r^{\prime}\right)$, as functions of $r^{\prime}$, get the asymptotic constant value

$$
c_{\varphi}=4 \pi \ln (2 L / R)
$$




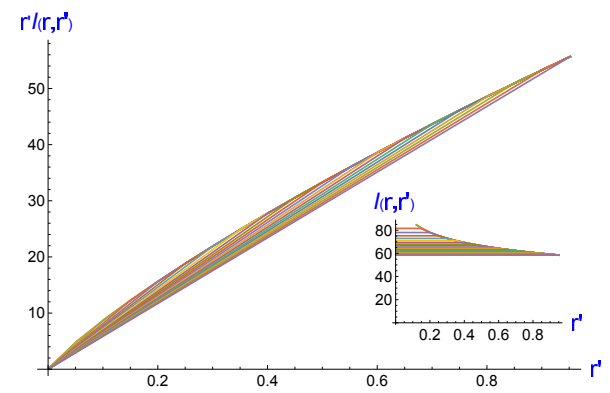

FIG. 1. Set of functions $r^{\prime} \mathscr{I}\left(r, r^{\prime}\right)$ versus $r^{\prime}$, for several values of $r$, the radial variables being scaled to the pipe radius. The insert displays the functions $\mathscr{I}\left(r, r^{\prime}\right)$ versus $r^{\prime}$, for given $r$. The $r$ values increase monotonically from the upper curve to the lower one $(r=0$ for the upper curve). $L / R=50$.

This relation can be derived by expanding (15) with respect to $a / L$, which gives $I\left(\varphi, r, r^{\prime}\right)=2 \ln 2-\ln (a / L)^{2}+O\left(a^{2} / L^{2}\right)$. As $r$ decreases towards zero, the curves $\mathscr{I}\left(r, r^{\prime}\right)$ versus $r^{\prime}$ do not remain constant, while keeping the asymptotic value $c_{\varphi}$ at $r^{\prime} / R=1$, see the insert in Fig.(1). However we have observed that approximating $\mathscr{I}\left(r, r^{\prime}\right)$ by the constant $c_{\varphi}$, for any $r$ values, yields a velocity profile which agrees very well with the exact calculation. This can be understood from the following argument. Ignoring the radial dependence of $u_{r^{\prime}}$, we note that the integrand of (14) contains the factor $r^{\prime}$. By looking at the curves $r^{\prime} \mathscr{I}\left(r, r^{\prime}\right)$ versus $r^{\prime}$ (for different $r$ values), one observes that they roughly merge with the linear function $c_{\varphi} r^{\prime}$, see the main curves in Fig.(b).

The next step to derive the radial profile, is to identify the solution of (14) with the solution of (12) for a circular pipe submitted to uniform pressure gradient in the $x$-direction. In cylindrical coordinates (12) becomes

$$
\frac{1}{r} \frac{\mathrm{d}\left(r \sigma_{x r}^{R e}(r)\right)}{\mathrm{d} r}+C=0,
$$

where $C=p_{x} / \rho$ is a constant, which will be taken positive in the following, and where the function $\sigma_{x r}^{R e}(r)$ is given by equation (14). A solution of (18) is

$$
\sigma_{x r}^{R e}(r)=-\frac{C}{2} r+\frac{A}{r},
$$

where $\mathrm{A}$ is a constant. On the axis of the pipe this solution diverges except for $\mathrm{A}=0$. However let us notice that the stress tensor must vanish at the surface of the pipe $r=R$, where the fluctuations normal to the surfaces vanish. Therefore the solution $\sigma_{x r}^{R e}(r)=-\frac{C}{2} r$ is not valid close to the pipe surface, where (18) has to include the viscous term $v \frac{\partial^{2} u}{\partial r^{2}}$ which was neglected above whereas it becomes of leading order in the inner boundary layer. In this domain (18) has to be replaced by

$$
\frac{1}{r} \frac{\partial}{\partial r} r\left(\sigma_{x r}^{R e}(r)-\rho v \frac{\partial u}{\partial r}\right)+C=0 .
$$

Escaping this boundary problem for the moment, the velocity profile of the flow has to obey the following equation in terms of the scaled variables $r / R=\hat{r}$ and $r^{\prime} / R=\hat{r}^{\prime}$

$$
\frac{C}{2} \hat{r}=K \rho\left|u_{\hat{r}}\right|^{1-\alpha} \int_{0}^{1} \mathrm{~d} \hat{r}^{\prime}\left|u_{, \hat{r}^{\prime}}\right|^{1+\alpha} \hat{r}^{\prime} \mathscr{I}\left(\hat{r}, \hat{r}^{\prime}\right)
$$

At zero order with respect to the approximation mentioned above, namely that $\mathscr{I}\left(r, r^{\prime}\right) \approx c_{\varphi}$ is independent of $r$, the solution of (21) is $u_{\hat{r}}^{(0)}(\hat{r})=k_{\alpha} \hat{r}^{1 /(1-\alpha)}$ with

$$
k_{\alpha}^{2}=\frac{3-\alpha}{1-\alpha} \frac{C}{2 K \rho c_{\varphi}} .
$$

Assuming $u(1)=0$ at the boundary of the pipe, the velocity field is given by

$$
u^{(0)}(\hat{r})=k_{u}\left(1-\hat{r}^{\xi}\right)
$$

where

$$
k_{u}=k_{\alpha} \frac{1-\alpha}{2-\alpha} \quad \xi=\frac{2-\alpha}{1-\alpha} .
$$

The profile is flatter and flatter as the exponent $\alpha$ approaches unity, see Fig.2(a). The good agreement between the zero order solution and the first order one is illustrated in Fig.2(b) where the first order solution is obtained by solving (21) with the correct functions $\mathscr{I}\left(\hat{r}, \hat{r}^{\prime}\right)$ and taking for $u_{, \hat{r}^{\prime}}$ (in the integrand) the $\hat{r}^{\prime}$-derivative of $u^{(0)}\left(\hat{r}^{\prime}\right)$. This result proves that although the expression of the Reynolds stress depends formally on the average velocity in a non local way, the non locality only changes the amplitude of the solution, since the approximation made above and justified by fig.(b), amounts to change (14) into $\sigma_{x r}^{R e}(r)=K^{\prime} \rho\left|u_{, r}(r)\right|^{1-\alpha}$, where the constant $K^{\prime}$ is equal to $K$ times an integral independent of $r$.

\section{B. Velocity profile close to the boundary}

Let us now return to the boundary condition for the averaged velocity field $u(r)$. From the way this stress was built one may guess that the boundary condition is the same as for a viscous fluid, namely that the velocity on the surface of the solid is the local velocity of this solid, which amounts to impose $u=0$ at the radius $r=R$ of the pipe. To take a physical case where the boundary condition on the bounding surface plays an important role, we recall the experimental work by Nikuradse ${ }^{14}$ in the nineteen thirties who reported a significant sensitivity of the turbulent friction with respect to a controlled roughness of the pipe wall. This is hard to explain if one assumes that turbulence is generated by large scale motion inflows decaying to small dissipative scales by non linear interaction. We note $\tau$ the tangential constraint,

$$
\tau(r)=\rho v \frac{\partial \bar{u}}{\partial r}-\sigma_{x r}^{R e}(r),
$$

which has to satisfy (20), or

$$
\frac{\partial}{\partial r}(r \tau(r))=C r
$$




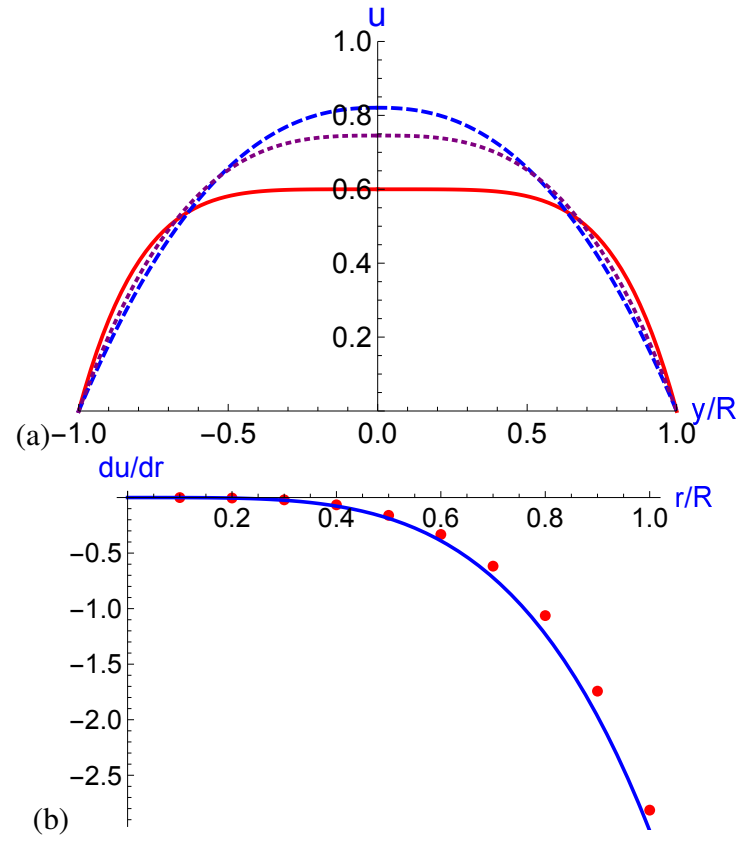

FIG. 2. (a)Profiles of the velocity along the diameter of the pipe for $\alpha=1 / 4$ in blue dashed line, $\alpha=1 / 2$ in purple dotted line, $\alpha=$ $3 / 4$ in red solid line, $L / R=50$ and the velocity is scaled to $\gamma=$ $\left(\frac{C}{2 K \rho c_{\varphi}}\right)^{1 / 2}$. (b) Solution of (21), derivative of the velocity versus $r / R$ for $\alpha=3 / 4$. The solid blue line displays the leading order solution (23) obtained with the approximation $\mathscr{I}\left(r, r^{\prime}\right) \approx c_{\varphi}$, the points are obtained by iteration at first order, only few of them are reported for clarity.

in the whole profile.

In the viscous domain, of width $\delta$ assumed to be much smaller than the radius, the solution of (26) at distance $z$ from the boundary, is $\tau(R-z)=\tau_{*}-C z$, where $\tau_{*}=\tau(R)$ is unknown for the moment. Let us precise that we consider the case of smooth surface, namely with roughness smaller than $\delta$. The tangential stress is supposed to be constant in the viscous layer, that implies $\delta<<\tau_{*} / C$. It follows that the solution for $z<\delta$ is

$$
u^{v i s}(z)=\tau_{*} z /(\rho v)
$$

which has to be linked for $z>\delta$ to the turbulent solution, of the form (23) up to a constant (see below). Note that the connection consists in relying two pieces of $u(r)$ with very different slopes, the linear solution of slope $u_{z}^{v i s}=\tau_{*} /(\rho v)$ and the turbulent solution of slope of order $U / R$, with $U$ the mean velocity in the turbulent domain. The ratio of these two slopes is equal to the Reynolds number. This is the familiar phenomenon of boundary layer: in the present case the socalled outer flow field is given by the solution of the integral equation. Near the wall, in the inner domain, viscosity comes into play and another approximation should be used. The two domains, inner and outer, should overlap in a common range of radii. In the present formulation of this question and contrary to other approaches to this question, we have at our disposal an explicit equation for the turbulent stress which yields therefore an also explicit boundary layer analysis whereas this problem is usually dealt within Prandtl or Prandtl-like approximation of the turbulent averages, which does not extend to the flow far from the wall (see recent work on this idea ${ }^{15}$ ). This outer region, which is in fact a transitory domain separating the inner viscous region from the flow far from the wall (often behaving like a plug flow), has been often described to obey a logarithmic law ${ }^{12}$, although its experimental observation remains a challenge due to the high Reynolds numbers needed ${ }^{16}$. So far, in our analytical results using the approximations explained above, we do not obtain clearly such logarithmic evolution. However, we want to argue that such transient behavior can mimic easily a logarithmic law as already discussed in turbulent channel flow models, so that the logarithmic behavior could be present in our model ${ }^{17}$. We plan in fact to publish a detailed analysis of the way the viscous boundary layer can be matched with the turbulent core of the pipe flow and to compare it with existing models and experimental results, particularly concerning the matching between the boundary layer and the solution in the core.

Specifically it is highly significant there to use the degree of freedom provided by the possible addition of an arbitrary uniform velocity field along the axis and outside of the viscous boundary layer.

\section{Navier condition}

In the theory presented here the sensitivity to surface roughness could follow from the substitution to the regular boundary condition of continuity of tangential velocity along a smooth wall by a so-called mixed condition of the Navier type like

$$
u_{t}+\lambda \frac{\partial u_{t}}{\partial n}=0
$$

where $u_{t}$ is the component of $u$ tangent to the surface, $u_{t}=u$ here and $n$ the coordinate locally normal to this surface, $n=z$ here. The quantity denoted as $\lambda$ has the physical dimension of a length. It represents, for a rough surface the r.m.s. of the fluctuations of height of the rough surface. With such a boundary condition, the solution of equation (18) for the Reynolds stress depends on $\lambda$. Because there is no length parameter in the problem besides geometrical parameter of the flow, like the diameter of pipes in Poiseuille flows, it could be that the final result, namely the drag per unit length of the pipe, depends sensitively on the length $\lambda$, as observed. This contrasts with the predictions of a theory based on the idea that large scale motion dominates dissipation by transfer of energy toward small scales: if one follows this idea it seems very hard to understand the sensitivity of observed dissipation to roughness of the boundaries, a roughness that changes small scales of the Euler dynamics only.

Let us point out that, contrary to the viscous case, all terms in the equation (12) for the balance of longitudinal momentum are quadratic with respect to the velocity. It is also important to point out that the mean velocity in equation (12) changes sign, as it should, when the pressure gradient driving the flow changes sign. In the case of a parallel flow in 
a pipe, the inertial stress $u_{i} u_{j}$ doesn't contribute to equation (12). By changing the sign of the velocity, Reynolds stress $\sigma_{i j}^{(R e)}$ changes sign.

\section{Divergence of integral in equation (10)}

As shown by equation (17) the integral over $X^{\prime}$ in equation (10) diverges logarithmically at large values of the coordinate $x^{\prime}$ along the direction of the pipe. Such a divergence is not unexpected because the integrand in this equation is proportional to the inverse of $\left|X^{\prime}-X\right|$ and so, if a component of $X^{\prime}$ does appear only there in the integrand, as is the case for $x^{\prime}$, the integral diverges. The same divergence would appear in any 2D geometry where the dimension perpendicular to the one of the flow is large. For instance in the case of a velocity flow in the $x$ direction bounded by a half plane $y=z=0, y$ and $z$ being the two other coordinates besides $x$. Therefore this logarithmic divergence seems to be quite common in situations where equation (13) could be pertinent. Indeed, as done in the previous section, one can avoid the divergence of the integral by considering a pipe with finite length $L$. However there is another way to get rid of this divergence by borrowing somehow ideas from quantum field theory, known to face logarithmic divergences and to get rid of them. This is done by "renormalizing" the coefficients of the bare theory. Without making a too close link between the two theories, one could view this "renormalization" (in the sense of Dirac and Heisenberg) as a way to get rid of the divergence by considering a coefficient $K$ tending to zero as $L$ diverges. Without being too accurate, this could amount to subtract from the right-hand side of equation (13) the same integral, but with $\left|X^{\prime}\right|$ instead of $\left|X^{\prime}-X\right|$. This is permitted according to the two principles lead out to derive this equation, (a) no arbitrary length scale is introduced in this way and (b) the basic symmetries, Galilean and rotational, are preserved. The result is the following integral equation for the renormalized Reynolds stress,

$$
\begin{aligned}
\sigma_{i j}^{R e}(X)= & K \rho|\nabla \times u(X)|^{1-\alpha} \int \mathrm{d} X^{\prime}\left|\nabla \times u\left(X^{\prime}\right)\right|^{\alpha} \\
& \times\left(\frac{1}{\mid\left(X-X^{\prime} \mid\right.}-\frac{1}{\left|X^{\prime}\right|}\right)\left(u_{i, j}+u_{j, i}\right)\left(X^{\prime}\right),
\end{aligned}
$$

This expression of the turbulent Reynolds stress can be used also for the turbulent Poiseuille flow, but now an upper length scale is not necessary. In this case the solution is still given by (21) but equation (15) is replaced by

$$
\begin{aligned}
I\left(\varphi, r, r^{\prime}\right)= & \int_{-\infty}^{+\infty} \mathrm{d} x^{\prime}\left(\frac{1}{\left(r^{2}+r^{\prime 2}-2 r r^{\prime} \cos (\varphi)+x^{\prime 2}\right)^{1 / 2}}\right. \\
& \left.-\frac{1}{\left(r^{\prime 2}+x^{\prime 2}\right)^{1 / 2}}\right)=\ln \left(\frac{a\left(r, r^{\prime}, \varphi\right)}{a\left(0, r^{\prime}, \varphi\right)}\right)^{2}
\end{aligned}
$$

which is independant of the pipe length. The integral in this expression converges, as expected. The integration over $\varphi$ of (30) is easier by using the variable $x=\cos \varphi$, it gives

$$
\mathscr{I}\left(r, r^{\prime}\right)=\int_{0}^{1} \mathrm{~d} x \frac{1}{\sqrt{1-x^{2}}} \ln \left(\left(1+\frac{r}{r^{\prime}}\right)^{2}+4 \frac{r}{r^{\prime}} x^{2}\right)
$$

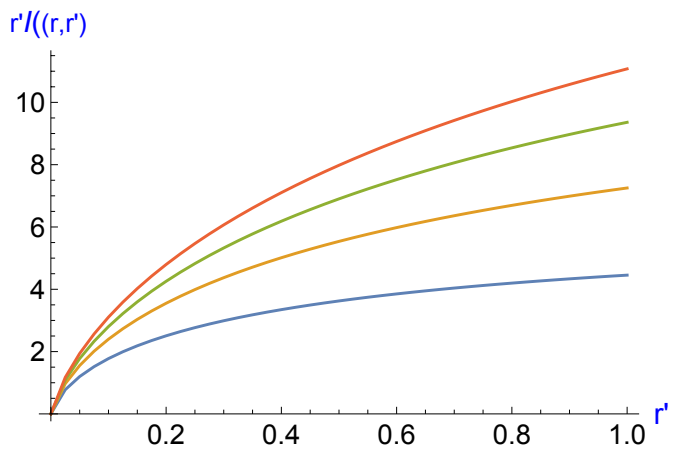

FIG. 3. Functions $r^{\prime} \mathscr{I}\left(r, r^{\prime}\right)$ versus $r^{\prime}$ for values of $r=$ $1 / 4,1 / 2,3 / 4,1$, from bottom to top. The radial variables are $\hat{r}, \hat{r}^{\prime}$ in the text, they are scaled to the pipe radius $R$.

The term $r^{\prime} \mathscr{I}\left(r, r^{\prime}\right)$ which is under the integral in equation (21) is plotted versus $r^{\prime}$ in Fig. 3, for several values of $r$, see captions. For a given value of $r$, this kernel is the difference between the top curve and the lower curve corresponding to the selected $r$ value in Fig.1. Note that the new function $\mathscr{I}\left(r, r^{\prime}\right)$ depends now on the variable $r$, although it was quite independent on $r$ in section III A ( $\mathrm{cf}$ the approximation made there).

Actually the new kernel corresponds to the term which have been neglected above when we derived the approximate expression (23) for the velocity profile $u(r)$. Nevertheless the numerical solution for this renormalized model of the stress tensor leads also (as in in section III A) to profiles $u(r)$ getting flatter and flatter as the exponent $\alpha$ approaches unity, as shown in Fig. 4. The solutions are obtained by iteration. Using the scaled velocity $u \rightarrow u / \gamma_{2}$ with $\gamma_{2}=\left(\frac{C}{2 K \rho}\right)^{1 / 2}$, equation (21) becomes

$$
\left|u_{, \hat{r}}\right|^{1-\alpha} \int_{0}^{1} \mathrm{~d} \hat{r}^{\prime}\left|u_{, \hat{r}^{\prime}}\right|^{\alpha} u_{, \hat{r}^{\prime}} \hat{r}^{\prime} \mathscr{I}\left(\hat{r}, \hat{r}^{\prime}\right) .=-\hat{r}
$$

At order zero we assume $u_{\hat{r}^{\prime}}^{(0)}=-\hat{r}^{\prime \beta}$, where $\beta$ is an adjustable exponent, see below. Inserting this expression inside the integral $I$ in the l.h.s. of (32), we get the first order solution $\left[u_{\hat{r}}^{(1)}\right]^{1-\alpha}=-\hat{r} I^{(0)}(1) / I^{(0)}(\hat{r})$, where the factor $I^{(0)}(1)$ ensures that $u_{\hat{r}}^{(1)}(1)=-1$. Then we repeat this procedure until the solution converges. We observed that the convergence arises very rapidly when using $u_{\hat{r}}^{(0)}=-\hat{r}$ for $\alpha$ of order $1 / 2$, and using $u_{, \hat{r}}^{(0)}=-\hat{r}^{3}$ for $\alpha$ close to unity, something obvious when looking at the profiles in Fig.4.

We plan to use this model (29) for the Reynolds tensor to solve other problems of turbulent flows.

\section{CONCLUSION AND PERSPECTIVES}

This work intended to explain how the centuries-old (exactly 333 years this year) law of drag by Newton can be used to illustrate that the dissipation occurs in fully developed turbulence by singular events, which are local in space and 


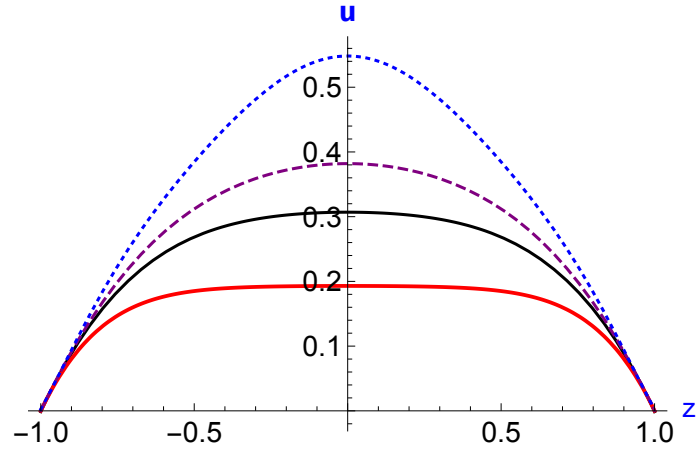

FIG. 4. Profiles of the velocity along the diameter of the pipe, for $\alpha=1 / 2,3 / 4,0.82,0.9$ from top curves to lower ones. The curves display $u(r)-u(1)$, with velocity scaled to $\gamma_{2}=\left(\frac{C}{2 K \rho}\right)^{1 / 2}$ and $z$ scaled to the pipe radius $R$.

time and described, until the time of blow-up, by solutions of the 3D Euler equations for incompressible fluids. A consequence of that is an explicit equation for the space dependent (but time averaged) velocity, resulting from an expression of Reynolds stress as a function of this average fluid velocity. Our approach differs from the classical approaches initiated by Boussinesq and Prandtl since no explicit turbulent viscosity is introduced ${ }^{12}$. The Reynolds stress tensor is therefore only determined by the mean flow field and witnesses the singularity of the inviscid flows because no extrinsic lengthscale is needed. This opens new perspectives. To take an example, the equation for the average velocity has a fairly nontrivial structure, being non linear, non local and non analytic. Therefore it could well be that, with given boundary conditions, it has more than one solution (a property not found in the exemple of Sec.III), as has its simpler counterpart for viscous fluid equations. This would fit well with the observation that turbulent flows may show bifurcations as reported by Coles ${ }^{18}$ in the case of circular Couette flows.

Another unexplained mystery in experimental turbulence is Toms effect ${ }^{19}$. This fairly spectacular phenomenon has long been observed. It displays an important drag reduction in turbulent flows (usually pipe flows) by the addition of minute amount of polymers in a concentration that does not change appreciably the regular viscosity of the fluid. Any explanation of this effect has to rely on the lessening of turbulent dissipation in such a flow. If dissipation is in singular events it could be that the rush to small scales in the corresponding self-similar solutions is stopped by polymers elongated in a more or less distorted state. This could stop the collapse process before its dissipative end. This strengthens the notion put forth by de Gennes ${ }^{19}$ that polymer additives suppress motions at the small length scales where the strain rates are high, cut- ting off the evolution of fluctuations toward small dissipative scales.

\section{ACKNOWLEDGEMENTS}

The authors thank Sergio Rica for useful discussions on the matter of this article.

The data that support the findings of this study are available from the corresponding author upon reasonable request.

${ }^{1}$ L. Landau, E.M. Lifshitz, Course of theoretical physics "Fluid Mechanics," (Pergamon, Oxford, 1987).

${ }^{2}$ U. Frisch, "Turbulence," ( Cambridge, Univ. Press, 1995).

${ }^{3}$ G. Falkovich. Fluid Mechanics (A short course for physicists). (Cambridge, University Press, 2011).

${ }^{4}$ F.G. Schmitt, ”About Boussinesq turbulent viscosity hypothesis: historical remarks and a direct evaluation of its validity", C. R. Mecanique 335, 617627 (2007).

${ }^{5}$ P. Sagaut and C. Cambon "Homogenous Turbulence Dynamics", (Cambridge Univ. Press, 2008).

${ }^{6} \mathrm{~J}$. Leray, "Essai sur le mouvement d'un fluide visqueux emplissant l'espace", Acta Math. 63, 193-248 (1934).

${ }^{7}$ Y. Pomeau, M. Le Berre and T. Lehner, "A case of strong non linearity: intermittency in highly turbulent flows", C.R. Mécanique. Paris, Special issue dedicated to Pierre Coullet, 347, 342 (2019). and ArXiv:1806.04893v2.

${ }^{8}$ Y. Pomeau and M. Le Berre, "Leray turbulence: what can we learn from acceleration compared to velocity", arXiv:1909.01724 (physics.flu-dyn)

${ }^{9} \mathrm{~K}$. Weierstrass, " On continuous functions of a real argument which possess a definite derivative for no value of the argument "'", Koniglich Preussichen Akademie der Wissenschaften, Mathematische Werke von Karl Weierstrass (Berlin, Germany: Mayer and Mueller, 1895), vol. 2, pp. 71-74.

${ }^{10} \mathrm{C}$. Josserand, Y. Pomeau and S. Rica, "Finite-time singularities as a mechanism for dissipation in a turbulent media", to appear in Phys.Rev. Fluids (2020); and arXiv:1910.05523v1(cond-math.stat-mech).

${ }^{11}$ A. de Chézy , "Formule pour trouver la vitesse de l'eau dans un conduit ou un canal de pente donnée ", Dossier 847, Ms 1915 of the manuscript collection of the Ecole nationale des Ponts et Chaussées, Paris; reproduced in Georges Mouret, Antoine Chézy: "Histoire d'une formule d'hydraulique", Annales des Ponts et Chaussées, Paris, 11e série, vol. 61, pp. 165-269 (1921).

${ }^{12}$ S.B. Pope, "Turbulent flows", (Cambridge Univ. Press, 2000).

${ }^{13}$ Y. Pomeau and M. Le Berre, "Scaling laws in turbulence", Arxiv:1912.12866-v1.

${ }^{14}$ J. Nikuradse, "Stromungsgesetz in rauhren Rohren" vDI Forshungsheft 361 (1933) [English transl. "Laws of flow in rough pipes", Technical report, NASA Technical Memorandum 1292. National Advisory Commission for Aeronautics, Washington, DC., 1950)] .

${ }^{15}$ G. Gioia and P. Chakraborty, "Turbulent Friction in Rough Pipes and the Energy Spectrum of the Phenomenological Theory", Phys. Rev. Lett. 96, 044502 (2006) ; see also N. Goldenfeld, "Roughness-Induced Critical Phenomena in a Turbulent Flow", Phys. Rev. Lett. 96, 044503 (2006).

${ }^{16}$ A.J. Smits, B.J. McKeon and I. Marusic "High-Reynolds Number Wall Turbulence", Ann. Rev. Fluid Mech. 43, 353-375 (2011)

${ }^{17}$ S. Chen, C. Foias, D.D. Holm, E. Olson, E.S. Titi and S. Wynne "CamassaHolm Equations as a Closure Model for Turbulent Channel and Pipe Flow", Phys. Rev. Lett. 81, 5338-5341 (1998).

${ }^{18}$ D. Coles, "Transition in circular Couette flow.", J. Fluid Mech. 21, 385 (1965).

${ }^{19}$ P-G de Gennes. "Introduction to Polymer Dynamics", (Cambridge University Press, New York, 1990). 